\title{
Analysis on the parameters of selected mapping without side information on PAPR performances
}

\begin{abstract}
Nowadays, the OFDM support the growing request of higher data rate transportation in both wired and wireless communication. The SLM technique is recognized as one of the most capable methods which are applied in OFDM system in order to reduce the problem of high PAPR. However the major drawback of SLM is transmission of side information which has negative result on data rate and bandwidth efficiency. Accordingly the SLM method without sending side information has been proposed. Therefore this paper investigates how the parameters in SLM techniques without Side Information (SI), could have effects on the PAPR performances of the method.
\end{abstract}

Keyword: CCDF; Extension factor; OFDM; PAPR; SLM 